\title{
Rings in which nilpotents form a subring
}

\section{JANEZ ŠTER}

\section{ABSTRACT.}

Let $R$ be a ring with the set of nilpotents $\operatorname{Nil}(R)$. We prove that the following are equivalent: (i) $\mathrm{Nil}(R)$ is additively closed, (ii) $\mathrm{Nil}(R)$ is multiplicatively closed and $R$ satisfies Köthe's conjecture, (iii) $\mathrm{Nil}(R)$ is closed under the operation $x \circ y=x+y-x y$, (iv) $\mathrm{Nil}(R)$ is a subring of $R$. Some applications and examples of rings with this property are given, with an emphasis on certain classes of exchange and clean rings.

Acknowledgements. The author would like to thank Nik Stopar for helpful discussions and Pace P. Nielsen for useful comments on the previous version of this paper.

\section{REFERENCES}

[1] Antoine, R., Nilpotent elements and Armendariz rings, J. Algebra, 319 (2008), No. 8, 3128-3140

[2] Ara, P., Extensions of exchange rings, J. Algebra, 197 (1997), No. 2, 409-423

[3] Călugăreanu, G., UU rings, Carpathian J. Math., 31 (2015), No. 2, 157-163

[4] Chen, W., On linearly weak Armendariz rings, J. Pure App. Algebra, 219 (2015), No. 4, 1122-1130

[5] Chun, Y., Jeon, Y. C., Kang, S., Lee, K. N. and Lee, Y., A concept unifying the Armendariz and NI conditions, Bull. Korean Math. Soc., 48 (2011), No. 1, 115-127

[6] Danchev, P. and Lam, T. Y., Rings with unipotent units, to appear in Publ. Math. Debrecen, 88 (2016)

[7] Diesl, A. J., Nil clean rings, J. Algebra, 383 (2013), 197-211

[8] Han, J., Jung, Y., Lee, Y. and Sung, H. J., Insertion-of-factors-property with factors nilpotents, Korean J. Math., 22 (2014), No. 4, 611-619

[9] Hirano, Y., Tominaga, H. and Yaqub, A., On rings in which every elements is uniquely expressible as a sum of a nilpotent and a certain potent element, Math. J. Okayama Univ., 30 (1988), 33-40

[10] Hong, C. Y., Kim, N. K., Lee, Y. and Nielsen, P., On $\sigma$-nil ideals of bounded index of $\sigma$-nilpotence, J. Algebra, 371 (2012), 492-509

[11] Koşan, T., Wang, Z. and Zhou, Y., Nil-clean and strongly nil-clean rings, J. Pure Appl. Algebra, 220 (2016), No. 2, 633-646

[12] Lam, T. Y., A first course in noncommutative rings, Graduate Texts in Mathematics 131, Springer-Verlag, New York, 2001

[13] Nielsen, P., Zentralblatt Review https :// zbmath.org/?q=an:1296.16016

[14] Smoktunowicz, A., Polynomial rings over nil rings need not be nil, J. Algebra, 233 (2000), No. 2, 427-436

UNIVERSITY OF LJUBLJANA

FACULTY OF MECHANICAL ENGINEERING

AŠKERČEVA 6, 1000 LJUBLJANA, SLOVENIA

E-mail address: janez.ster@fs.uni-lj.si

Received: 05.10.2015; In revised form: 29.12.2015; Accepted: 05.01.2016

2010 Mathematics Subject Classification. 16N40, 16 U99.

Key words and phrases. Nilpotent, NR ring, Armendariz ring, exchange ring, strongly nil clean ring. 